\title{
A Note on the Riccati Equation
}

\author{
R. Cruz-Santiago ${ }^{1,} *$ J. López-Bonilla1, J. Morales² \\ ${ }^{1}$ ESIME- Zacatenco, Instituto Politécnico Nacional, Col. Lindavista CP 07738, México DF \\ ${ }^{2}$ CBI-Área de Física AMA, Universidad Autónoma Metropolitana-Azcapotzalco, Av. San Pablo 180, CP 02200, México \\ * jlopezb@ipn.mx
}

\begin{abstract}
We show the relationship between the solutions of two Riccati equations associated to Schrödinger equations interconnected via a Darboux transformation.
\end{abstract}

Keywords: riccati equation, schrödinger equation, darboux transformation, energy spectrum in quantum mechanics

\section{INTRODUCTION}

The Schrödinger equation permits to study a quantum system via the wave function, besides this equation leads to the energy levels for a given potential. Then it is natural to ask if this quantum information can be employed for another system with a different potential but with the same energy spectrum. The answer is yes if both systems are interconnected through a Darboux transform, and in this process the Riccati equation has an important role. Therefore, if two Schrödinger equations have a Darboux type relationship then it is useful to investigate the behaviour of the corresponding nonlinear Riccati equations. Our analysis shows that a Darboux transformation adds an exact derivative to the Riccati solution, and we consider that this fact explains why the energy values are invariant under Darboux mapping.

\section{SCHRÖDINGER AND RICCATI EXPRESSIONS}

The Schrödinger equation:

$$
\Psi^{\prime \prime}+(\lambda-\mathrm{u}) \Psi=0,
$$

where $\Psi, \mathrm{u}$ and $\lambda$ represent the wave function, the potential and the eigenvalue of energy, respectively, can be transformed to a Riccati equation (name introduced by D'Alembert in 1763), Riccati 1724 , Kryachko 2005:

$$
\mathrm{R}+\mathrm{R}^{2}+\lambda-u=0
$$

employing the following change in the dependent variable, Young 1931, Lanczos 1997, Kryachko 2005:

$\mathrm{R}=\frac{\Psi^{\prime}}{\Psi}$

If now a Darboux transformation, Darboux 1882,
Matveev and Salle 1991, López-Bonilla, Morales and Ovando 2002, Caltenco, López-Bonilla and Acevedo 2004, is applied to (1) then a Schrödinger equation with the same energy levels, but with different wave functions $\Phi$ and another potential $U$, is obtained:

$\Phi^{\prime \prime}+(\lambda-\mathrm{U}) \Phi=0$

such that:

$\Phi=\Psi^{\prime}-\Psi \sigma_{1}, \quad \mathrm{U}=u-2 \sigma_{1}^{\prime}, \quad \sigma_{1}=\frac{\Psi_{1}^{\prime}}{\Psi_{1}}$,

being $\Psi_{1}$ the solution of (1) for $\lambda_{1}$ :

$\Psi_{1}^{\prime \prime}+\left(\lambda_{1}-\mathrm{u}\right) \Psi_{1}=0$

Then (4) has associated the Riccati equation:

$\tilde{\mathrm{R}}^{\prime}+\tilde{\mathrm{R}}^{2}+(\lambda-U)=0 \quad, \quad \tilde{\mathrm{R}}=\frac{\Phi^{\prime}}{\Phi}$,

and it is natural to ask on the relationship between $\mathrm{R}$ and $\tilde{R}$.

From (5):

$\Phi=\Psi^{\prime}-\Psi \sigma_{1} \stackrel{(3)}{=} \Psi \mathrm{R}-\Psi \sigma_{1}=\Psi\left(\mathrm{R}-\sigma_{1}\right)$,

which can be used in (7):

$\tilde{\mathrm{R}}=\frac{1}{\Psi\left(\mathrm{R}-\sigma_{1}\right)}\left[\left(\mathrm{R}-\sigma_{1}\right) \Psi^{\prime}+\Psi\left(\mathrm{R}-\sigma_{1}\right)^{\prime}\right]=\frac{\Psi^{\prime}}{\Psi}+\frac{\left(\mathrm{R}-\sigma_{1}\right)^{\prime}}{\mathrm{R}-\sigma_{1}}$,

and thus to obtain an interesting expression between the Riccati functions:

$\tilde{\mathrm{R}}=\mathrm{R}+\frac{d}{d} \mathrm{~h} \quad\left(\mathrm{R}-\sigma_{1}\right)$,

when the $\epsilon_{\Perp 1}^{d x}$ associated Schrödinger equations are connected via a Darboux transformation. The 
relations:

$$
\mathrm{R}^{\prime \prime}+2 \mathrm{R} \mathrm{R}^{\prime}=\sigma_{1}{ }^{\prime \prime}+2 \sigma_{1} \sigma_{1}^{\prime}=u^{\prime},
$$

permit to verify that (8) into (7) leads to (2).

The following diagram illustrates our procedure:

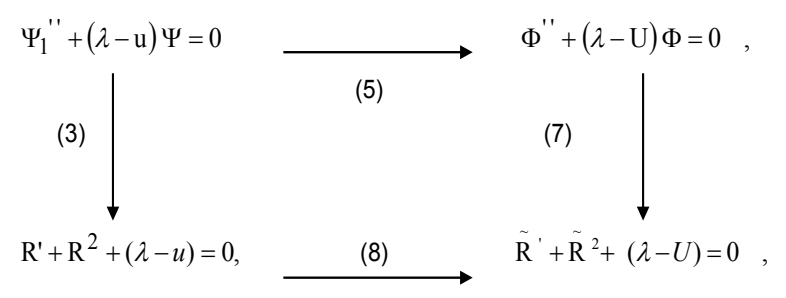

Therefore, (8) represents the corresponding Darboux transformation for the Riccati equation in its normal form.

\section{CONCLUSION}

The expression (8) exhibits that an arbitrary Darboux mapping adds an exact derivative to the Riccati solution, which may be a underlying reason for the invariance of energy spectrum in the Schrödinger equation, being $\operatorname{Ln}\left(\mathrm{R}-\sigma_{1}\right)$ a generating function for Darboux transform in the Riccati scheme.

\section{REFERENCES}

[1] Caltenco, J.H., López-Bonilla, J., and Acevedo, M., 2004. A comment on the Darboux transformation. Acta Acad. Paed. Agriensis, Sectio Mathematicae 31: 121-123

[2] Darboux, G., 1882. Sur une proposition relative aux équations linéaires. Comptes. Rend. Acad. Sci. 94: 1456-1459

[3] Kryachko, E.S., 2005. Notes on the Riccati equation, Collect. Czech. Chem. Commun. 70: 941-950

[4] Lanczos, C., 1997. Linear differential operators, Dover, New York, pp. 370

[5] López-Bonilla, J., Morales, J., and Ovando, G., 2002. Darboux transformations and isospectral potentials in quantum mechanics. Apeiron 9: 20-25

[6] Matveev, V.B., and Salle, M.A., 1991. Darboux transformations and solitons, Springer-Verlag, Berlin, pp. 7

[7] Riccati, J., 1724. Actorum eruditorum quae lipsiae publicantur Suppl. 8: 66 -73

[8] Young, L.A., 1931. A note on local momentum in wave mechanics. Phys. Rev. 38: 1612-1614 